\title{
SEVERE MICROBIAL KERATITIS IN TEMPERATE AND TROPICAL WESTERN AUSTRALIA
}

\author{
ANNETTE GEBAUER ${ }^{1}$, CHARLES N. J. McGHEE ${ }^{1,3}$ and GEOFFREY J. CRAWFORD ${ }^{1,2}$ \\ Perth, Australia; and Sunderland
}

\begin{abstract}
SUMMARY
To determine the patterns of severe microbial keratitis in Western Australia, all acute admissions over a 2 year period to the Department of Ophthalmology, Royal Perth Hospital were assessed. Fifty-three cases $(n=53)$ of severe, presumed microbial keratitis were identified. Seventy per cent of these eyes had a visual acuity of $6 / 60$ or less on admission and only $38.8 \%$ had $6 / 12$ or better corrected acuity following resolution of the keratitis. The most commonly identified predisposing factors were: prior ocular surgery with or without exposed monofilament sutures $(43.4 \%)$; contact lens wear $(22.6 \%)$; lid malposition $(17.0 \%)$; history of ocular trauma $(15.1 \%)$; and history of previous herpes simplex keratitis $(13.2 \%)$. It is notable that $26.4 \%$ of the subjects had been applying topical ophthalmic corticosteroids prior to admission. Following corneal scrape or biopsy a positive microbial diagnosis was made in $71 \%$ of samples, with Gram-negative and Gram-positive bacterial isolates being equally frequent. Five cases of Acanthamoeba keratitis were identified following corneal biopsy. Where antibiotic sensitivities were available, it was noted that $61.5 \%$ of Grampositive and $46.1 \%$ of Gram-negative bacteria were susceptible to chloramphenicol, with $84.6 \%$ of Gramnegative bacteria being sensitive to gentamicin. Many of these severe cases of microbial keratitis might have been avoided, or their severity lessened, by earlier identification of predisposing risk factors, more intensive and appropriate antibiotic administration, and improved patient education following ocular surgery.
\end{abstract}

Severe infectious keratitis can be a debilitating disease which affects all age groups and can result in severe

From: ${ }^{1}$ Department of Ophthalmology, Royal Perth Hospital, Wellington Street, Perth, 6001 Western Australia; ${ }^{2}$ Lions Eye Institute, 2 Verdun Street, Nedlands, Perth, 6009 Western Australia; ${ }^{3}$ Corneal Diseases \& Excimer Laser Unit, Sunderland Eye Infirmary, Queen Alexandra Road, Sunderland SR2 9HP, UK.

Correspondence to: Professor C. N. J. McGhee, University of Dundee Department of Ophthalmology, Ninewells Hospital and Medical School, Dundee DD1 9SY, UK. pain, prolonged therapy, and significant, permanent impairment of visual acuity. ${ }^{1-7}$ We retrospectively reviewed all acute admissions for severe, presumed microbial keratitis over a 2 year period to Royal Perth Hospital, Western Australia. This is a major 700 bed teaching hospital with a tertiary ophthalmic referral service which serves a very large catchment area (2.5 million $\mathrm{km}^{2}$ ), including the metropolitan and country areas of Western Australia. The 1.54 million residents live in varied climatic conditions from the cool temperate south to the tropical conditions of north Western Australia. This unique combination of very varied climatic and living conditions within the vast catchment area of one ophthalmic unit results in a confusing array of clinical presentations of keratitis, and by analysing these cases the authors' aims were: to detect local ocular factors which might predispose to and enhance the severity of microbial keratitis; to determine the course and clinical outcome of these cases; and to identify elements of clinical management which might be improved in order to lessen the morbidity of this condition.

\section{SUBJECTS AND METHODS}

Royal Perth Hospital provides a specialised ophthalmology service which attracts approximately 13000 outpatient reviews and 1250 inpatient admissions per year. During the period January 1991 to January 1993 there were more than 2600 inpatient admissions, among which 53 cases of presumed severe microbial keratitis were identified. These cases were retrospectively reviewed and outcome determined over a follow-up period of 3-18 months. Associated ocular conditions such as marginal keratitis, herpes zoster ophthalmicus keratitis, and sterile corneal peripheral infiltrates were excluded from further study. The following were recorded for each identified case: age, place of origin; duration of symptoms before admission; predisposing local ocular and systemic factors; visual acuity on admission and final review; ulcer size; presence of hypopyon; perforation; recent topical

Eye (1996) 10, 575-580 (C) 1996 Royal College of Ophthalmologists 
Table I. Factors predisposing to microbial keratitis

\begin{tabular}{lc}
\hline Predisposing factors & $\begin{array}{c}\text { No. of patients } \\
(n=53)\end{array}$ \\
\hline Ocular surgery & $23(43.4 \%)$ \\
Cataract extraction \pm IOL & 7 \\
Penetrating keratoplasty & 8 \\
Other procedures & 8 \\
Contact lens wear & $12(22.6 \%)$ \\
Lid malposition & 9 \\
Trauma & 8 \\
HSV keratitis & 7 \\
Dry eye disease & 2 \\
Band/bullous keratopathy & 5 \\
\hline
\end{tabular}

Factors identified in 53 consecutive eyes admitted with severe microbial keratitis which might have predisposed these eyes to, or enhanced the severity of, microbial keratitis.

ophthalmic treatment; organisms cultured; antimicrobial sensitivities of organisms cultured; definitive ophthalmic treatment; and surgical procedures performed. In cases in which no organisms were identified, and clinical improvement did not occur in the presence of fortified antibiotic treatment, corneal biopsies were performed 48 hours after cessation of all topical medication.

All patients admitted by the corneal service routinely have a corneal 'scrape' performed on admission utilising a sterile hypodermic needle, sterile spatula or scalpel blade. Specimens are provided for Gram staining and material from corneal scrapes is inoculated directly onto the following media at the time of collection: "chocolate agar' (CA) plates (GC Agar base, Oxoid Australia, West Heidelberg, Victoria, Australia) plus 1\% Isovitalex (BBL Microbiology Systems, Cockeysville, $\mathrm{MD}$ ); and a Sabouraud's dextrose agar (SAB) plate (Oxoid Australia) containing $50 \mathrm{mg} / \mathrm{l}$ chloramphenicol. Scalpel blades were placed in serum broth (SB) (5\% horse serum in No. 2 nutrient broth; Oxoid Australia), whilst swabs were placed in Amies charcoal transport media (Disposable Products, Technology Park, South Australia) and inoculated onto the following within 15 hours of collection: a blood agar plate (BA), a CA plate and a SAB plate. $\mathrm{BA}$ and CA plates were incubated at $37^{\circ} \mathrm{C}$ in $8 \%$ $\mathrm{CO}_{2}$ for 48 hours and SB was incubated at $37^{\circ} \mathrm{C}$ aerobically for 4 days with subculture onto $\mathrm{CA}$ after 4 days, or earlier if turbid. SAB plates were incubated at $30^{\circ} \mathrm{C}$ aerobically for 4 days, then at room temperature for 4 weeks.

In addition, specimens from suspected cases of Acanthamoeba keratitis were transported in saline and plated onto water agar plates with bacterial suspension and biopsy specimens were prepared for transmission electron microscopy. Specimens were also processed for the identification of herpes viruses.

\section{Clinical Presentation}

\section{RESULTS}

A total of 29 males (55\%) and 24 females (45\%)
Table II. Topical therapy at time of admission

\begin{tabular}{lc}
\hline Topical drug & $\begin{array}{c}\text { No. of patients } \\
(n=39)\end{array}$ \\
\hline Antibiotics & $37(94.9 \%)$ \\
Chloramphenicol & 25 \\
Tobramicin & 7 \\
Gentamicin & 2 \\
Other & 3 \\
Corticosteroid & $14(35.8 \%)$ \\
Antivirals & $6(15.4 \%)$ \\
Miscellaneous & $7(17.9 \%)$ \\
\hline
\end{tabular}

Topical ophthalmic agents being applied to 39 of 53 eyes presenting with severe microbial keratitis $(n=53)$. Within the subgroup of eyes receiving topical medication $(n=39)$, notably 14 $(35.9 \%)$ were applying topical corticosteroids. Interestingly, 14 eyes $(26.4 \%)$ were on no topical medication at the time of presentation.

were admitted to Royal Perth Hospital with suspected severe microbial keratitis during the period studied. Eighty-three per cent of the patients were Caucasians resident in metropolitan urbanised areas; the remaining 9 patients came from country towns, including 4 Aborigine patients from tropical northwest Western Australia and a further Aborigine patient from the inland wheatbelt.

Patient age at presentation ranged from 19 to 95 years $(60.4 \pm 22.7$ years, mean $\pm \mathrm{SD})$, with more than $45 \%$ of the patients being 61 years of age or older. Almost half of these patients $(47.2 \%)$ had experienced symptoms of keratitis or ocular infection for less than 2 weeks prior to admission, although it is notable that $11.3 \%$ actually had symptoms of more than 2 months' duration and in all of these latter cases extensive outpatient treatment and investigation had failed to yield a pathogen susceptible to prescribed treatment prior to referral. Seventy per cent of these patients $(n=37)$ had a presenting visual acuity of less than or equal to 6/60 (all visual acuities expressed represent best spectacle corrected visual acuities). Factors which might predispose subjects to microbial keratitis were present in the majority of cases $(94.3 \%)$ and are highlighted in Table I. Thirtynine of these patients (73.6\%) were on some form of topical ophthalmic preparation at the time of admission, and of those prescribed topical medication, $94.9 \%$ were applying an antibiotic preparation and $35.9 \%$ were using a corticosteroid preparation (Table II).

\section{Ocular Features}

In 8 cases the size of the ulcer was not noted accurately or was unrecordable (2 cases of perforation and expulsion). In keratitis cases where accurate measurements were available $(n=45)$, only 13 $(28.8 \%$ ) were small ulcers (less than $2 \mathrm{~mm}$ in diameter); the remainder were greater than $2 \mathrm{~mm}$ diameter, with 8 eyes $(17.8 \%)$ demonstrating an infected lesion greater than $5 \mathrm{~mm}$ in at least one diameter. Fifteen patients $(28.3 \%)$ exhibited hypo- 
Table III. Surgical procedures performed

\begin{tabular}{lc}
\hline Operation & $\begin{array}{c}\text { No. of patients } \\
(n=22)\end{array}$ \\
\hline Evisceration/enucleation & 4 \\
Corneal biopsy & 5 \\
Vitrectomy & 2 \\
Penetrating keratoplasty & 2 \\
Intravitreal antibiotics & 1 \\
Synechiolysis & 1 \\
Suture removal & 3 \\
Entropion repair & 4 \\
\hline
\end{tabular}

Surgical procedures required upon 53 patients admitted with severe microbial keratitis.

pyon at presentation. Corneal perforation at presentation was noted in 3 patients. One of these $3 \mathrm{had}$ a history of trauma, a corneal laceration and a 7 day delay in seeking medical treatment; in 2 other cases corneal perforation was associated with expulsion of intraocular contents (following a history of chronic ulceration of several weeks' duration in one, and an associated long-standing history of carcinoma of the cornea in the other). Descemetocoeles were noted in 3 cases, 1 of which had obviously perforated but sealed without surgical intervention. The majority of patients $(83 \%)$ had an inpatient episode of 7-10 days' duration, though 9 patients $(17 \%)$ were admitted for longer than 14 days and 3 admissions exceeded 28 days.

\section{Microbiology}

Corneal scrapes and/or biopsies were performed in 48 of the 53 patients admitted. In 2 patients with corneal perforation scrapes were not performed, while in 1 other case poor patient cooperation (dementia) prevented the procedure; however, in 2 subjects noted to have very small ulcers the reason for omission of a corneal scrape was unstated. A positive microbial result, by Gram stain, culture or histopathology (biopsy), was recorded in 34 of the 48 subjects, providing a positive yield of $70.8 \%$. These 34 subjects yielded 47 positive specimens, with bacteria identifiable in 41 specimens (29 subjects). Gram-positive and Gram-negative organisms were identified with almost equal frequency $(51.2 \%$ vs $48.8 \%)$. The most commonly detected organisms were Staphylococcus species (41.5\%), Staphylococcus aureus $(14.6 \%)$, coagulase-negative staphylococci (14.6\%) and Pseudomonas species (12.2\%). Acanthamoeba species were identified by corneal biopsy in 5 cases, and only 1 microbiologically confirmed case of herpes simplex was identified.

\section{Antibiotic Sensitivities and Antimicrobial Regimes}

A total of 13 Gram-negative and 13 Gram-positive cultured bacteria were assessed for susceptibility to antibiotics (see above). The majority of Grampositive bacteria were susceptible to chloramphenicol $(61.5 \%)$, with only $23.1 \%$ being susceptible to cephalosporin antibiotics; thus 10 of the 13 Gram- positive bacterial isolates $(76.9 \%)$ were sensitive to either chloramphenicol or a cephalosporin. The majority of Gram-negative bacteria were sensitive to either gentamicin $(84.6 \%)$ or less commonly chloramphenicol $(46.1 \%)$. In total 12 of the 13 Gram-negative isolates were sensitive to gentamicin or chloramphenicol, with the remaining case being sensitive to tobramycin. Interestingly, $57.7 \%$ of bacterial infections demonstrated in vitro sensitivity to the antibiotic being prescribed at presentation.

Whilst awaiting the results of microbiological sensitivity testing, all patients with presumed bacterial keratitis were commenced upon fortified hourly antibiotics in the form of guttae gentamicin $1.5 \%$ and guttae cephalothin 5\%. Presumed herpes simplex keratitis was treated with oc. acyclovir and in presumed acanthamoebal infections a combination of oc. propamidine isethionate (Brolene) and guttae neosporin was employed. Topical antimicrobial regimes were subsequently adjusted in the light of microbiological sensitivities and clinical response.

\section{Surgical Intervention}

Twenty-two patients underwent some form of surgical intervention in relation to the diagnosis, treatment, or rehabilitation of the affected eye. These are listed in Table III.

\section{Visual Outcome}

Seventy per cent of eyes $(n=37)$ had a visual acuity of $6 / 60$ or less on admission and, of these, 4 eyes were lost (3 eviscerations, 1 enucleation), and 13 patients were lost to long-term follow-up for geographic or other logistical reasons. At latest follow-up (mean 7.8 months, range 3-18 months post-admission), of 36 eyes available for such long-term review only 14 eyes $(38.8 \%)$ had corrected visual acuities of $6 / 12$ or better, whereas 14 eyes (38.8\%) had poorer than $6 / 60$ vision due to severe scarring, anterior segment inflammatory changes or the effect of endophthalmitis.

\section{DISCUSSION}

Due to the very large catchment area of Royal Perth Hospital, to which patients are referred from up to $1800 \mathrm{~km}$ away, the size of the region itself can produce unique management problems. In particular many of the aboriginal patients from the north of the state are often far removed from medical facilities and therefore late presentation of eye disorders is not uncommon. Indeed, all aboriginal patients in this study had visual acuity in the affected eye of less than $6 / 60$ on presentation and because of subsequent scarring or endophthalmitis this level of visual disability was not improved following treatment and resolution of the keratitis. It is also notable that in these cases of delayed presentation multiple 
organisms were grown on bacterial culture of corneal scrapes, thus requiring prolonged hospitilisation and intensive topical therapy. Unfortunately the difficulties of maintaining sterility of ophthalmic drugs in a village setting with temperatures of $40{ }^{\circ} \mathrm{C}$ or more, and the limited access of health visitors, far less ophthalmologists, means that early local rehabilitation of these patients is difficult.

Long periods of hospitalisation have profound financial implications. In this present study the median inpatient admission period was 7-10 days, although 9 patients $(17 \%)$ were admitted for more than 15 days and 3 patients had a hospital stay exceeding 1 month. It is entirely conceivable that by early, appropriate management, such inpatient periods might be reduced, since, in most cases in which organisms were isolated, these organisms were actually found to be sensitive to the first-line $(57.7 \%)$ or second-line $(80.7 \%)$ antibiotics being utilised. Therefore an earlier detection/presentation of the keratitis coupled with a more intensive topical regime (rather than simple q.d.s. regimes) might have reduced the severity of the keratitis, thus reducing or avoiding the period of admission.

We have demonstrated an extremely wide age distribution in the present series, whereas other authors have demonstrated a more marked bimodal distribution, with Musch et al. ${ }^{2}$ attributing this phenomenon to accidental trauma in the younger age group. Interestingly, in the present study trauma is rivalled by contact lens wear as a predisposing risk factor in patients under 30 years of age. Hypopyon was noted in $40 \%$ of confirmed Gram-positive ulcers and all Pseudomonas ulcers in the present study. Ormerod et al. ${ }^{1}$ have previously noted a very strong corelation between hypopyon and Streptococcus pneumoniae keratitis, trauma and age greater than 60 years, but a less marked association of hypopyon with Pseudomonas aeruginosa keratitis. It is notable that large corneal ulcers greater than $5 \mathrm{~mm}$ diameter were relatively common $(17.8 \%)$ in the present study and significant acute ocular morbidity in the form of frank corneal perforation $(n=3)$ or descemetocoele formation $(n=3)$ occurred in $11.3 \%$ of eyes reviewed.

Predisposing ocular risk factors for microbial keratitis were identified in $94.3 \%$ of our patients, with several having multiple risk factors. The most common risk factors appeared to be previous ocular surgery $(43.4 \%)$, contact lens wear $(22.6 \%)$, abnormal lid position $(17.0 \%)$, trauma $(15.1 \%)$, previous herpetic keratitis (13.2\%) and long-term ophthalmic corticosteroid application $(11.3 \%)$. Others have noted trauma, ${ }^{2}$ including surgical trauma, as the most frequent risk factor in microbial keratitis, whereas Coster et al. ${ }^{7}$ found the most frequent predisposing factors were past HSV keratitis and trauma in younger patients. Fortunately traumatic cases, predominantly in younger patients, tend to present early, ${ }^{2}$ and in the present study 8 of 9 such patients regained $6 / 12$ or better vision in the affected eye with the exception of 1 case of late presentation. From the results presented and those of other studies $^{1-7}$ one might surmise that a wet, smooth corneal epithelium which has not been exposed to the risks of contact lens wear or the iatrogenic effects of an ophthalmic surgeon ${ }^{8,9}$ is actually the best safeguard against developing infectious keratitis. ${ }^{10-17}$

The largest single group of predisposing factors in this series was previous ocular surgery $(43.4 \%)$, and a very significant proportion of these patients had pathology related to corneal sutures. Although some patients with broken sutures will present early with foreign body sensation, ${ }^{9}$ routine removal of sutures might avoid the unacceptably high morbidity from protruding monofilament sutures. ${ }^{8,9}$ Patients with penetrating keratoplasty constituted more than $30 \%$ of the patients who had undergone previous surgery in this series, and due to multiple risks such as suturerelated problems, graft failure, topical corticosteroids and graft denervation, the incidence of microbial keratitis after penetrating keratoplasty can be surprisingly high with a frequency of $1.8-4.9 \% .^{18,19}$ By regular clinical review, removal of sutures prior to degradation or loosening, $8,9,20$ controlled topical steroid use and patient education regarding the risks and signs of keratitis, this distressingly high complication rate might be reduced.

We recorded 5 cases of Acanthamoeba keratitis associated with cosmetic contact lens wear in previously healthy corneas. In recent years it has become increasingly apparent that Acanthamoeba keratitis is strongly associated with contact lens wear and poor lens hygiene. ${ }^{21-25}$ Despite increasing awareness of this problem, diagnosis is often delayed; and even when the diagnosis is suspected these organisms are not readily detected by routine microbiological means. ${ }^{11,21-25}$ In the 5 cases presented 4 had an incorrect preliminary diagnosis of herpes simplex keratitis and had been unsuccessfully treated by prolonged but inadequate topical regimes prior to referral. In large series the most common misdiagnosis of Acanthamoeba keratitis is HSV keratitis (70-76\%), though earlier diagnosis is becoming much more common due to increased awareness of this disease. ${ }^{25,26}$ In the present study, despite multiple corneal scrapes and attempts to grow the organism on bacterial seeded culture plates, all diagnoses were eventually made on histological examination of corneal biopsy specimens. ${ }^{26,27}$

Pseudomonas aeruginosa colonisation of contact lenses and lens cases has previously been associated with keratitis associated with contact lens wear, ${ }^{16,28-30}$ possibly due to increased adherence of 
these organisms to contact lenses, particularly soft lenses. ${ }^{31,32}$ In this study 2 of 4 cases of Pseudomonas keratitis were associated with contact lens wear and a further case demonstrated both Acanthamoeba and Pseudomonas from a single corneal biopsy. In all cases of severe keratitis samples of any bottles of topical medication, contact lenses and lens cases in current use should also be cultured. ${ }^{3}$

A large number of patients in this study required surgical intervention in the form of diagnostic biopsy or more extensive surgical procedures including penetrating keratoplasty. Unfortunately despite surgical intervention eyes were still lost, including 3 cases of evisceration and 1 of enucleation for a blind painful eye. Penetrating keratoplasty per se did not feature largely in this study, in that the eyes which developed perforation had such extensive intraocular involvement that no visual recovery was anticipated. Indeed, Kirkness et al. ${ }^{33}$ have noted that emergency penetrating keratoplasty in microbial keratitis results in only a $51 \%$ graft survival at 5 years compared with a $90 \%$ survival when the microbial keratitis is completely settled on vigorous medical therapy and the cornea allowed to heal prior to elective intervention.

Certain groups had a poorer prognosis depending upon the organisms involved. Although all eyes with Acanthamoeba keratitis had a presenting visual acuity of $6 / 60$ or less, 4 of 5 eyes achieved $6 / 12$ spectacle corrected visual acuity at follow-up. However, the majority of eyes which yielded multiple organisms on culture presented with visual acuity less than $6 / 60$ and all remained so despite treatment.

The proportion of culture-negative cases varies in large studies from $15 \%$ to $63 \% .^{1,7.34-38}$ In the present study organisms were isolated in 29 of 48 eyes on the basis of corneal scrape and culture $(60.4 \%)$ and in another 5 patients diagnosis was confirmed following corneal biopsy (10.4\%), providing an overall positive microbiology result in $70.8 \%$ of cases analysed. Compared with other Australian studies, similar percentages of Gram-positive bacterial isolates but slightly greater numbers of Gram-negative isolates were identified. ${ }^{7,38}$ As in these other studies, we noted Staphylococcus aureus and Pseudomonas to be the most commonly isolated Gram-positive and Gram-negative bacteria respectively. ${ }^{7,38}$ Surprisingly, we identified no cases of fungal keratitis. With the wide range of climatic conditions pertaining in Western Australia, from the temperate south to the tropical north, one might expect a variation in cultures obtained, and in fact cases from the northern areas exhibited a greater incidence of mixed and multiple isolates on culture. The reasons for the failure to isolate responsible organisms in $36 \%$ of corneal scrapes are complex, but must include: pretreatment with antibiotics; failure to stop anti- biotics 24-48 hours prior to rescrape; ${ }^{39}$ type of topical anaesthetic agent used, with perhaps nonpreserved benoximate being most suitable; ${ }^{40}$ inadequate specimen or choice of media; sterile or noninfectious corneal ulceration; and atypical organisms such as Acanthamoeba. In the present study $92.3 \%$ of Gram-negative organisms isolated were sensitive to either gentamicin or chloramphenicol and $77 \%$ of Gram-positive organisms were sensitive to cephalosporins or chloramphenicol. Intensive topical fortified gentamicin and cephalothin were the drugs of choice until full microbial sensitivities were available (although currently an alternative regime of monotherapy with a fluoroquinolone antibiotic might be considered).

The short- and long-term ocular morbidity from severe microbial keratitis is very significant, and in the present series almost half the patients required a hospital admission of more than 10 days. Despite vigorous antimicrobial therapy and repeated attempts to achieve microbial identification, almost $40 \%$ of eyes demonstrated a final visual acuity of $6 / 60$ or less and a further 4 were eviscerated or enucleated. Nineteen patients required some form of diagnostic or therapeutic surgical intervention. Several predisposing risk factors were identified, the most striking being previous ocular surgery, suture-related infection and contact lens wear. Poor prognostic factors include: multiple organism isolates, delay in presentation, visual acuity of less than $6 / 60$ on presentation, and the prior use of topical corticosteroids. With persistently negative cultures, in cases of painful keratitis, the possibility of Acanthamoeba keratitis must be noted and corneal biopsy considered. However, based on the preceding observations it would appear that several factors might logically be modified (where possible) to reduce the incidence and severity of microbial keratitis, including: regular review and patient education of subjects with corneal grafts; routine removal of all corneal sutures at 9 months to 1 year in cases of cataract surgery; more intensive (increased frequency) initial antibiotic therapy; early correction of lid malpositions, especially in the dry eye; and further public education with regard to the risks of poor contact lens hygiene.

Key words: Microbial keratitis, Ocular surgery, Contact lens, Ocular trauma, Corticosteroid, Acanthamoeba.

\section{REFERENCES}

1. Ormerod LD, Hertzmark E, Gomez D, et al. Epidemiology of microbial keratitis in Southern California. Ophthalmology 1987;94:1322-33.

2. Musch DC, Sugar A, Meyer RS. Demographic and predisposing factors in corneal ulceration. Arch Ophthalmol 1983;101:1545-8.

3. Schein OD, Waffon PJ, Boruchoff SA, Kenyon KR. Microbial keratitis associated with contaminated ulcer medications. Am J Ophthalmol 1988;105:361-5. 
4. Fong LP, Ormerod LD, Kenyon KR, Foster CS. Microbial keratitis complicating penetrating keratoplasty. Ophthalmology 1988;95:1269-75.

5. Bates AK, Kirkness CM, Ficker LA, et al. Microbial keratitis after penetrating keratoplasty. Eye 1990;4:748.

6. Ormerod LD, Fong LP, Foster CS. Corneal infection in mucosal scarring disorders and Sjögren's syndrome. Am J Ophthalmol 1988;105:512-8.

7. Coster DJ, Badenoch PR. Host, microbial and pharmacological factors affecting the outcome of suppurative keratitis. Br J Ophthalmol 1987;71:96-101.

8. Acheson JS, Lyons CJ. Ocular morbidity due to monofilament nylon sutures. Eye 1991;5:106-12.

9. Danjoux JP, Reck AC. Corneal sutures: is routine removal really necessary? Eye 1994;8:339-42.

10. Ormerod LD, Smith RE. Contact lens associated microbial keratitis. Arch Ophthalmol 1986;104:79-83.

11. Theodore FH, Jakobiec SA, Juechter KB, et al. The diagnostic value of a ring infiltrate in acanthamoebic keratitis. Ophthalmology 1985;92:1471-79.

12. Donzis PB, Mondino BJ, Weissman BA, Bruckner DA. Microbial analysis of contact lens tear systems contaminated with Acanthamoeba. Am J Ophthalmol 1989;108:53-6.

13. Schein OD, Glynn RJ, Poggio EC, et al. The relative risk of ulcerative keratitis among users of daily wear and extended wear soft contact lenses. N Engl J Med 1989;321:773-8.

14. Kent HD, Cohen EJ, Laibson PR, Arentsen JJ. Microbial keratitis and corneal ulceration associated with therapeutic soft contact lenses. CLAO J 1990;16: 49-52.

15. Chalupa E, Swabrick HA, Holden BA, Sjostrand J. Severe corneal infections associated with contact lens wear. Ophthalmology 1987;94:17-22.

16. Aswad M, John T, Barza M, Kenyon K, Baum J. Bacterial adherence to extended wear soft contact lenses. Ophthalmology 1990;97:296-302.

17. Bailey CS. A review of relative risks associated with four types of contact lenses. Cornea 1990;9:S59-61.

18. Lamensdorf M, Wilson LA, Waring GO, Cavanagh HD. Microbial keratitis after penetrating keratoplasty. Ophthalmology 1982;89 (Suppl):124

19. Tuberville AW, Wood TO. Corneal ulcers in corneal transplants. Curr Eye Res 1981;1:479-84.

20. Heaven CJ, Davison CRN, Cockcroft PM. Bacterial contamination of nylon corneal sutures. Eye 1995;9: 116-8.

21. Moore MB, McCulley JP. Acanthamoeba keratitis associated with contact lenses. Br J Ophthalmol 1989; 73:271-5.
22. Moore MB. Acanthamoeba keratitis and contact lens wear: the patient is at fault. Cornea 1990;9:S33-5.

23. Nauherm RC, Brockman RJ, Stopak SS, et al. Survival of Acanthamoeba in contact lens rinse solutions. Cornea 1990;9:290-3.

24. Larkin DF, Kilvington S, Easty DL. Contamination of contact lens storage cases by Acanthamoeba and bacteria. Br J Ophthalmol 1990;74:133-5.

25. Bacon AS, Frazer DG, Dart JKG, et al. A review of 72 consecutive cases of Acanthamoeba keratitis 19841992. Eye 1993;7:719-25.

26. Tay Kearney ML, McGhee CNJ, Crawford GJ. Acanthamoeba keratitis: a masquerade presentation in six cases. Aust NZ J Ophthalmol 1993;21:237-45.

27. Lee T, Green WR. Corneal biopsy. Ophthalmology 1990;97:718-21.

28. Dart JK. Predisposing factors in microbial keratitis: significance of contact lens wear. $\mathrm{Br} \mathrm{J}$ Ophthalmol 1988;72:926-30.

29. Cooper RL, Constable RJ. Infective keratitis in self contact lens wearers. Br J Ophthalmol 1977;61:250-4.

30. Schein OD, Ormerod LD, Barraquer E, et al. Microbiology of contact lens related keratitis. Cornea 1989;8: 281-5.

31. Klotz SA, Misara RP, Butrus SI. Contact lens wear enhances adherence of Pseudomonas aeruginosa and binding of lectins to the cornea. Cornea 1990;9:266-70.

32. Stern GA. Pseudomonas keratitis and contact lens wear: the lens/eye is at fault. Cornea 1990;9:S36-8.

33. Kirkness CM, Ficker LA, Steele A, Rice NS. The role of penetrating keratoplasty in the management of microbial keratitis. Eye 1991;5:425-31.

34. Perry LD, Brinser JH, Kolodner H. Anaerobic corneal ulcers. Ophthalmology 1982;89:636-42.

35. Ormerod LD. Causation and management of microbial keratitis in South Tropical Africa. Ophthalmology 1987;94:1662-8.

36. Liesegang TJ, Forster RK. Spectrum of microbial keratitis in Southern California. Am J Ophthalmol 1980;90:38-47.

37. Asvell P, Stenson S. Ulcerative keratitis: survey of thirty years' laboratory experience. Arch Ophthalmol 1982;100:77-80.

38. McClellan KA, Bernard PJ, Billson FA. Microbial investigations in keratitis at the Sydney Eye Hospital. Aust NZ J Ophthalmol 1989;17:413-6.

39. Ficker L, Kirkness C, McCartney A, Seal D. Microbial keratitis: the false negative. Eye 1991;5:549-59.

40. Badenoch PR, Coster DJ. Antimicrobial activity of topical anaesthetic preparations. $\mathrm{Br} \mathrm{J}$ Ophthalmol 1982;66:364. 